\title{
Simultaneous Diagonal Flips in Plane Triangulations*
}

\author{
Prosenjit Bose $\quad$ Jurek Czyzowicz ${ }^{\ddagger} \quad$ Zhicheng Gao $\quad$ Pat Morin ${ }^{\S}$ \\ David R. Wood"
}

October 31, 2018

\begin{abstract}
Simultaneous diagonal flips in plane triangulations are investigated. It is proved that every $n$-vertex triangulation with at least six vertices has a simultaneous flip into a 4-connected triangulation, and that it can be computed in $\mathcal{O}(n)$ time. It follows that every triangulation has a simultaneous flip into a Hamiltonian triangulation. This result is used to prove that for any two $n$-vertex triangulations, there exists a sequence of $\mathcal{O}(\log n)$ simultaneous flips to transform one into the other. The total number of edges flipped in this sequence is $\mathcal{O}(n)$. The maximum size of a simultaneous flip is then studied. It is proved that every triangulation has a simultaneous flip of at least $\frac{1}{3}(n-2)$ edges. On the other hand, every simultaneous flip has at most $n-2$ edges, and there exist triangulations with a maximum simultaneous flip of $\frac{6}{7}(n-2)$ edges.
\end{abstract}

keywords: graph, plane triangulation, diagonal flip, simultaneous flip, Hamiltonian

\section{Introduction}

A (plane) triangulation is a simple planar graph with a fixed (combinatorial) plane embedding in which every face is bounded by a triangle (that is, a 3-cycle). So that we can speak of the interior and exterior of a cycle, one face is nominated to be the outerface, although the choice of outerface is not important for our results.

Let $v w$ be an edge of a triangulation $G$. Let $(v, w, x)$ and $(w, v, y)$ be the faces incident to $v w$. Then $x$ and $y$ are distinct vertices, unless $G=K_{3}$. We say that $x$ and $y$ see $v w$. Let $G^{\prime}$ be the embedded graph obtained from $G$ by deleting $v w$ and adding the edge $x y$, such that in the cyclic order of the edges incident to $x$ (respectively, $y$ ), $x y$ is added between $x v$ and $x w$ ( $y w$ and $y v$ ).

\footnotetext{
$\S$ School of Computer Science, Carleton University, Ottawa, Canada ( $\{$ jit,morin\}@scs . carleton.ca).

‡Département d'informatique et d'ingénierie, Université du Québec en Outaouais, Gatineau, Canada (Jurek.Czyzowicz@uqo.ca).

ฯ School of Mathematics and Statistics, Carleton University, Ottawa, Canada (zgao@math. carleton.ca).

"Departament de Matemàtica Aplicada II, Universitat Politècnica de Catalunya, Barcelona, Spain (david.wood@upc.edu). Research partially completed at Carleton University and McGill University (Montréal).

${ }^{*}$ A preliminary version of this paper was published in the Proceedings of the 17th Annual ACM-SIAM Symposium on Discrete Algorithms (SODA '06). Research of the Canadian authors is supported by the Natural Sciences and Engineering Research Council of Canada (NSERC). Research of D. Wood is supported by the Government of Spain grant MEC SB2003-0270, and by the projects MCYT-FEDER BFM2003-00368 and Gen. Cat 2001SGR00224.
} 
If $G^{\prime}$ is a triangulation, then $v w$ is (individually) flippable, and $G$ is flipped into $G^{\prime}$ by $v w$. This operation is called a (diagonal) flip, and is illustrated in Figure 1 If $G^{\prime}$ is not a triangulation and $G \neq K_{3}$, then $x y$ is already an edge of $G$; we say that $v w$ is blocked by $x y$, and $x y$ is a blocking edge.

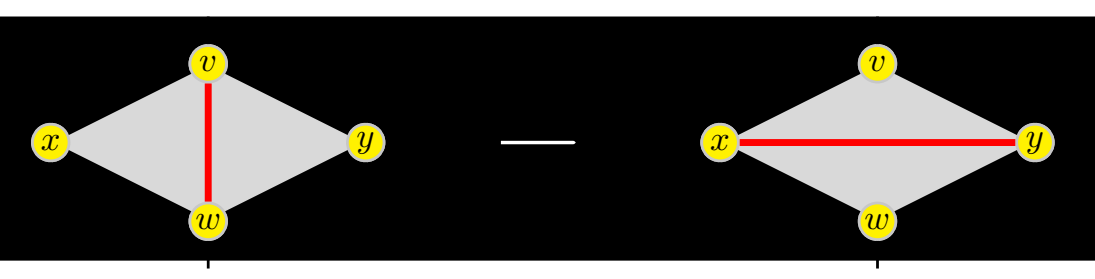

Figure 1: Edge $v w$ is flipped into $x y$.

In 1936, Wagner [35] proved that a finite sequence of diagonal flips transform a given triangulation into any other triangulation with the same number of vertices. Since then diagonal flips in plane triangulations [11, 12, 13, 16, 17, 20, 21, 23, 24, 26, 33] and in triangulations of other surfaces [3, 7, 8, 18, 22, 24, 25, 26, 27, 36] have been studied extensively. It can be shown that for triangulation with $n$ vertices, the number of flips in Wagner's proof is $\mathcal{O}\left(n^{2}\right)$. Komuro [16] improved this bound to $\mathcal{O}(n)$. The best known bound is $\max \{6 n-30,0\}$ due to Mori et al. [20].

For labelled triangulations, Sleator et al. [33] proved that $\mathcal{O}(n \log n)$ flips are sufficient to transform one labelled triangulation with $n$ vertices into any other, and $\Omega(n \log n)$ flips are sometimes necessary. The upper bound was independently rediscovered by Gao et al. [11]. Note that the above-mentioned $\mathcal{O}(n)$ upper bound in the unlabelled setting [16, 20] can also be obtained by a careful analysis of the proof by Sleator et al. [33].

Wagner [35] in fact proved that every $n$-vertex triangulation can be transformed by a sequence of flips into the so-called standard triangulation $\Delta_{n}$, which is illustrated in Figure 2 and is defined as the triangulation on $n$ vertices with two dominant vertices (adjacent to every other vertex). Clearly two $n$-vertex triangulations each with two dominant vertices are isomorphic. To transform one $n$-vertex triangulation $G_{1}$ into another $G_{2}$, first transform $G_{1}$ into $\Delta_{n}$, and then apply the flips to transform $G_{2}$ into $\Delta_{n}$ in reverse order. A similar approach is used in this paper in the context of simultaneous flips in triangulations.

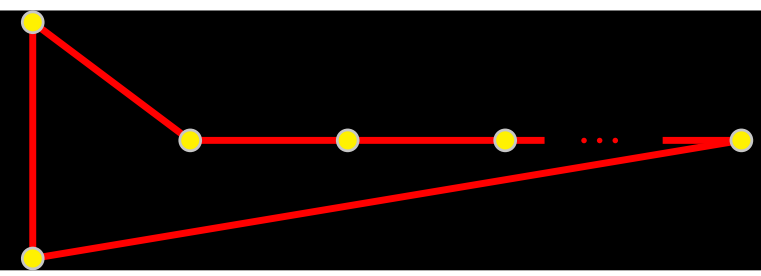

Figure 2: The standard triangulation and a Hamiltonian cycle.

Let $S$ be a set of edges in a plane triangulation $G$. The embedded graph obtained from $G$ by flipping every edge in $S$ is denoted by $G\langle S\rangle$. If $G\langle S\rangle$ is a triangulation, then $S$ is (simultaneously) flippable in $G$, and $G$ is flipped into $G\langle S\rangle$ by $S$. This operation is called a simultaneous (diagonal) flip. Note that it is possible for $S$ to be flippable, yet $S$ contains non-flippable edges, and it is 
possible for every edge in $S$ to be flippable, yet $S$ itself is not flippable. As far as the authors are aware, simultaneous flips have previously been studied only in the more restrictive context of geometric triangulations of a point set [10]. Individual flips have also been studied in a geometric context [14, 15].

In Section 2 we characterise flippable sets and give a number of introductory lemmas. Our first main result states that every triangulation with at least six vertices can be transformed by one simultaneous flip into a 4-connected (and hence Hamiltonian) triangulation. Moreover, this flip can be computed in $\mathcal{O}(n)$ time for $n$-vertex triangulations. These results are presented in Section 3 In Section 4 we study simultaneous flips in maximal outerplanar graphs. We prove that for any two $n$-vertex maximal outerplanar graphs, there exists a sequence of $\mathcal{O}(\log n)$ simultaneous flips to transform one into the other. The method used is the basis for the main result in Section 5 which states that for any two $n$-vertex triangulations, there exists a sequence of $\mathcal{O}(\log n)$ simultaneous flips to transform one into the other. This result is optimal for many pairs of triangulations. For example, if one triangulation has $\Theta(n)$ maximum degree and the other has $\mathcal{O}(1)$ maximum degree, then $\Omega(\log n)$ simultaneous flips are needed, since one simultaneous flip can at most halve the degree of a vertex. This also holds for diameter instead of maximum degree. Finally in Section 6 the maximum size of a simultaneous flip is studied. It is proved that every triangulation has a simultaneous flip of at least $\frac{1}{3}(n-2)$ edges. On the other hand, every simultaneous flip has at most $n-2$ edges, and there exist triangulations with a maximum simultaneous flip of $\frac{6}{7}(n-2)$ edges.

\section{Basics}

We start with a characterisation of flippable sets that is used throughout the paper. Two edges of a triangulation that are incident to a common face are consecutive. If two consecutive edges are simultaneously flipped, then the two new edges cross, as illustrated in Figure 3(a). Thus no two edges in a flippable set are consecutive. Two edges form a bad pair if they are seen by the same pair of vertices. If a bad pair of edges are simultaneously flipped, then the two new edges are parallel, as illustrated in Figure 3(b). Thus no two edges in a flippable set form a bad pair. If an edge $v w$ is blocked by an edge $p q$ as illustrated in Figure 3(c), then $v w$ is not individually flippable, but $v w$ can be in a flippable set $S$ as long as $p q$ is also in $S$. We now show that these three properties characterise flippable sets.

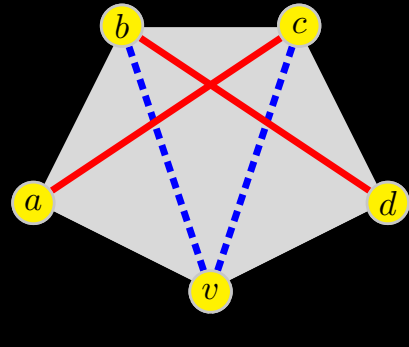

(a)

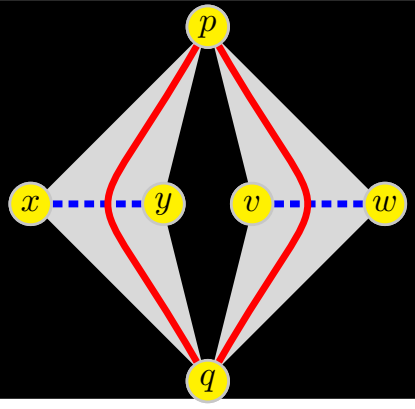

(b)

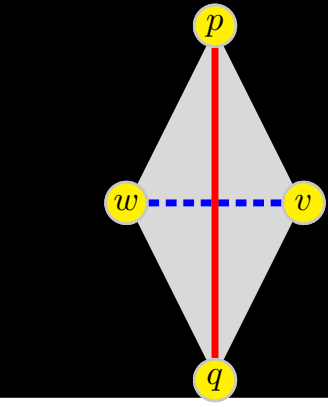

(c)

Figure 3: Obstacles to a flippable set. Dashed edges are flipped to create bold edges. Shaded regions are faces 
Lemma 2.1. A set of edges $S$ in a triangulation $G \neq K_{3}$ is flippable if and only if:

(1) no two edges in $S$ are consecutive,

(2) no two edges in $S$ form a bad pair, and

(3) for every edge $v w \in S$, either $v w$ is flippable or the edge that blocks $v w$ is also in $S$.

Proof. We have already seen that each condition is necessary for $S$ to be flippable. Now suppose that all three conditions are satisfied. Since no two edges in $S$ are consecutive, $G\langle S\rangle$ is a graph embedded in the plane and every face is a triangle. Suppose that two edges $e_{1}$ and $e_{2}$ are parallel in $G\langle S\rangle$. Since $G$ has no parallel edges, $e_{1}$ and $e_{2}$ are both not in $G$. If exactly one of $e_{1}$ and $e_{2}$ is in $G$, then condition (3) fails. If neither of $e_{1}$ and $e_{2}$ are in $G$, then the edges in $S$ that flipped to $e_{1}$ and $e_{2}$ form a bad pair.

Note that condition (1) in Lemma 2.1 is equivalent to saying that the edges of the dual $G^{*}$ that correspond to $S$ form a matching. (The dual $G^{*}$ of $G$ is the plane graph with one vertex for every face of $G$, such that two vertices of $G^{*}$ are adjacent whenever the corresponding faces in $G$ are incident to a common edge.)

A cycle $C$ in a triangulation $G$ is separating if deleting the vertices of $C$ from $G$ produces a disconnected graph.

Lemma 2.2. An edge in a separating triangle $T$ of a triangulation is individually flippable.

Proof. Consider an edge $v w$ in $T$. Say $v w$ is seen by $p$ and $q$. Then one of $p$ and $q$ is inside $T$, and the other is outside $T$. Thus $p q$ is not an edge, and $v w$ is flippable.

The next observation quickly follows from the Jordan Curve Theorem.

Lemma 2.3. Let $v w$ be an edge of a triangulation that is seen by vertices $p$ and $q$. Suppose that $p$ is inside some cycle $C$ and $q$ is outside $C$. Then $v w \in C$.

The next two results show that blocking edges are nearly always flippable, and except for essentially one case, do not appear in a bad pair.

Lemma 2.4. A blocking edge is individually flippable in a triangulation $G \neq K_{4}$.

Proof. Let $v w$ be an edge of $G$ that is blocked by $p q$. Without loss of generality, $w$ is inside the triangle $p v q$. If $p v q$ is a separating triangle, then $p q$ is flippable by Lemma 2.2. If $p v q$ is not separating, then $p w q$ must be a separating triangle since $G \neq K_{4}$. Therefore, $p q$ is flippable by Lemma 2.2

Lemma 2.5. Suppose that $v w$ and $x y$ are a bad pair in a triangulation $G$, both seen by vertices $p$ and $q$. Suppose that vw blocks some edge ab. Then $x y$ and $a b$ are consecutive, and vw and $x y$ are in a common triangle (amongst other properties).

Proof. Without loss of generality, $w$ and $x$ are inside the cycle $(v, p, y, q)$, and $b$ is inside the triangle $(v, a, w)$, as illustrated in Figure 4 Now $(v, p, y, q)$ is a separating 4-cycle with $w$ in its interior. Since $w$ is adjacent to $a$ and $b$, both $a$ and $b$ must be on the boundary of $(v, p, y, q)$. It follows that $p=b$ and $y=a$. If $w \neq x$, then the neighbours $w$ and $a$ are respectively on the inside and outside of the cycle $(p, x, q, v)$, which is not possible. Thus $w=x$. Hence $x y$ and $a b$ are consecutive, and $v w$ and $x y$ are in a common triangle. 


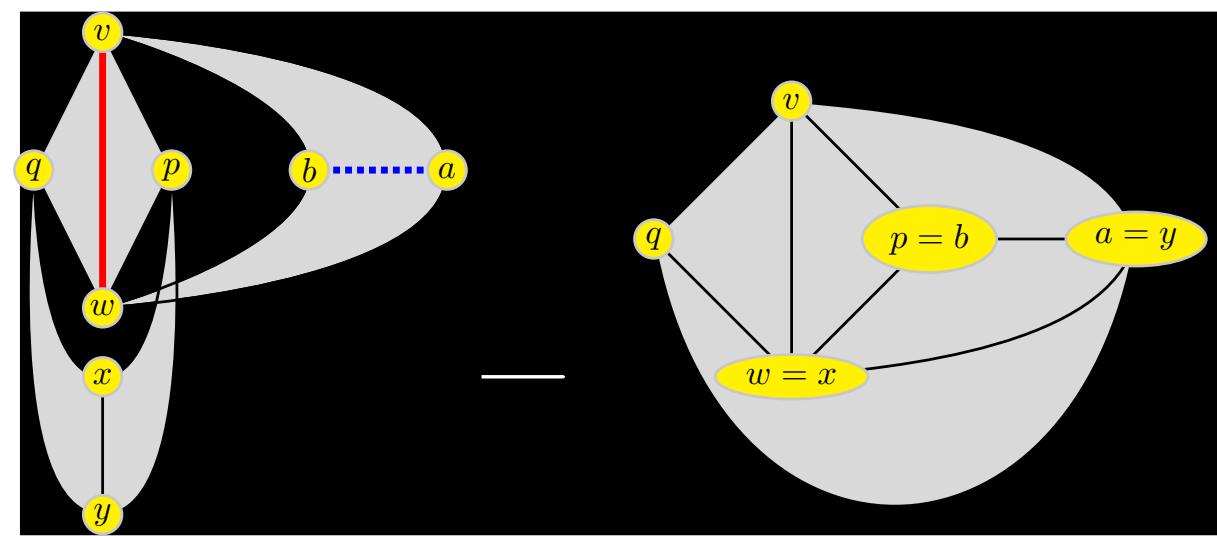

Figure 4: The only case when a blocking edge $v w$ is in a bad pair.

\section{Flipping into a 4-Connected Triangulation}

The main result in this section is that every triangulation with at least six vertices has a simultaneous flip into a 4-connected (and hence Hamiltonian) triangulation. It is well known that a triangulation is 4-connected if and only if it has no separating triangle. Thus our focus is on flips that break separating cycles.

Lemma 3.1. Let $S$ be a set of edges in a triangulation such that no two edges of $S$ are in a common triangle, and every edge in $S$ is in a separating triangle. Then $S$ is flippable.

Proof. By Lemma 2.2, every edge in $S$ is individually flippable. Thus, by Lemma2.1, it suffices to prove that no two edges in $S$ form a bad pair. Suppose that $v w, x y \in S$ form a bad pair. Then $v w$ and $x y$ are seen by the same pair of vertices $p$ and $q$. Let $T$ be a separating triangle containing $v w$. Then one of $p$ and $q$ is inside $T$, and the other is outside $T$. By Lemma $2.3 x y$ must be an edge of $T$, which implies that $v w$ and $x y$ are in a common triangle. This contradiction proves that there is no bad pair of edges both in $S$, and $S$ is flippable.

Lemma 3.2. Let $G$ be a triangulation with $n \geq 6$ vertices. Let $S$ be a set of edges in $G$ that satisfy the conditions in Lemma 3.1 and suppose that every separating triangle contains an edge in $S$. Then $G\langle S\rangle$ is 4-connected.

Proof. Suppose on the contrary, that $G\langle S\rangle$ contains a separating triangle $T=(u, v, w)$. Let $S^{\prime}$ be the set of edges in $G\langle S\rangle$ that are not in $G$. We proceed by case-analysis on $\left|T \cap S^{\prime}\right|$ (refer to Figure 5). Since every separating triangle in $G$ has an edge in $S,\left|T \cap S^{\prime}\right| \geq 1$.

Case 1. $\left|T \cap S^{\prime}\right|=1$ : Without loss of generality, $v w \in S^{\prime}, u v \notin S^{\prime}$, and $u w \notin S^{\prime}$. Suppose $x y$ was flipped to $v w$. Then $x y$ is in a separating triangle $x y p$ in $G$. Any vertex adjacent to both $v$ and $w$ must be a vertex of the separating triangle xyp. Thus $p=u$. Since $G$ has at least six vertices, at least one of the triangles $\{(u, v, x),(u, v, y),(u, w, x),(u, w, y)\}$ is a separating triangle. Thus at least one of the edges in these triangles is in $S$. Since $x y \in S$, and no two edges of $S$ appear in a common triangle, $\{u x, u y, v x, v y, w x, w y\} \cap S=\emptyset$. Thus $u v$ or $u w$ is in $S$. But then $u v w$ is not a triangle in $G\langle S\rangle$, which is a contradiction.

Case 2. $\left|T \cap S^{\prime}\right|=2$ : Without loss of generality, $u v \in S^{\prime}, v w \in S^{\prime}$, and $u w \notin S^{\prime}$. Suppose $x y$ was flipped to $u v$, and $r s$ was flipped to $v w$. Without loss of generality, $y$ and $s$ are inside $u v w$ 


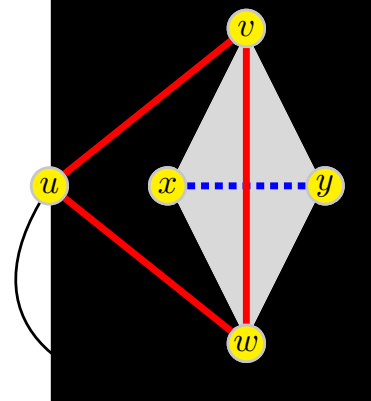

Case 1

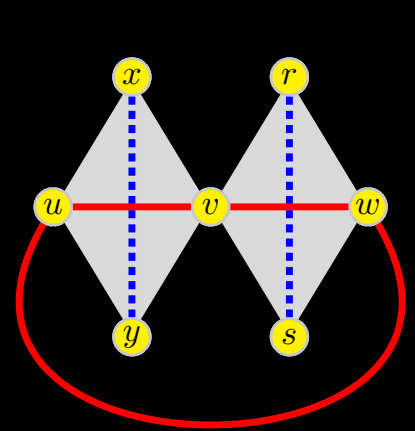

Case 2

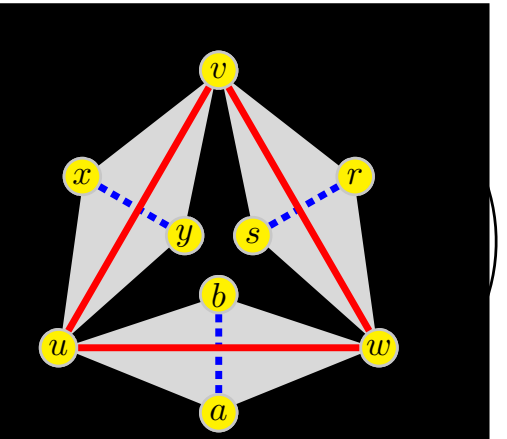

Case 3

Figure 5: Dashed edges are flipped to create a bold separating triangle. Shaded regions are faces.

in $G\langle S\rangle$. Then in $G, x y$ was in a separating triangle $x y z$, and $r s$ was in a separating triangle rst. By an argument similar to that in Lemma 3.1 $z=w$ and $t=u$. But then the subgraph of $G$ induced by $\{u, v, w, x, y, r, s\}$ is not planar, or it contains parallel edges in the case that $x=r$ and $y=s$.

Case 3. $\left|T \cap S^{\prime}\right|=3$ : Suppose $x y$ was flipped to $u v, r s$ was flipped to $v w$, and $a b$ was flipped to $u w$. Without loss of generality, $y, s$ and $b$ are inside $u v w$ in $G\langle S\rangle$. In $G, x y$ was in a separating triangle $x y z, r s$ was in a separating triangle $r s t$, and $a b$ was in a separating triangle $(a, b, c)$. By an argument similar to that in Lemma 3.1 $z=w, t=u$, and $c=v$. But then the subgraph of $G$ induced by $\{u, v, w, x, y, r, s, a, b\}$ is non-planar, or contains parallel edges in the case that $y=s=b$ and $x=r=a$.

In each case we have derived a contradiction. Therefore $G\langle S\rangle$ has no separating triangle, and thus is 4-connected.

Observe that the restriction in Lemma 3.2 to triangulations with at least six vertices is unavoidable. Every triangulation with at most five vertices has a vertex of degree three, and is thus not 4-connected.

We now consider how to determine a set of edges that satisfy Lemma 3.2

Lemma 3.3. Let $e$ be an edge of an $n$-vertex triangulation $G$. Then $G$ has a set of edges $S$ that can be computed in $\mathcal{O}(n)$ time such that $e \in S$ and every face of $G$ has exactly one edge in $S$.

Proof. Biedl et al. [1] proved the following strengthening of Petersen's matching theorem [30]: every 3-regular bridgeless planar graph has a perfect matching that contains a prespecified edge and can be computed in linear time. The dual $G^{*}$ is a 3-regular bridgeless planar graph with $2 n-4$ vertices. A perfect matching in $G^{*}$ corresponds to the desired set $S$.

Lemma 3.3 only accounts for triangles of $G$ that are faces. We account for separating triangles as follows.

Lemma 3.4. Let $e$ be an edge of an n-vertex triangulation $G$. Then $G$ has a set of edges $S$ such that $e \in S$ and every triangle of $G$ has exactly one edge in $S$. 
Proof. We proceed by induction on the number of separating triangles. The result follows for a triangulation with no separating triangles by Lemma 3.3. Now suppose $G$ has $k>0$ separating triangles, and the lemma holds for triangulations with less than $k$ separating triangles. Let $T$ be a separating triangle of $G$. Let the components of $G \backslash T$ have vertex sets $V_{1}$ and $V_{2}$. Consider the induced subgraphs $G_{1}:=G\left[V_{1} \cup T\right]$ and $G_{2}:=G\left[V_{2} \cup T\right]$. Without loss of generality, the given edge $e$ is in $G_{1}$. Both $G_{1}$ and $G_{2}$ have less than $k$ separating triangles. By induction $G_{1}$ has a set of edges $S_{1}$ such that $e \in S_{1}$, and every triangle of $G_{1}$ has exactly one edge in $S_{1}$. Let $e_{2}$ be the edge in $S_{1} \cap T$. By induction, $G_{2}$ has a set of edges $S_{2}$ such that $e_{2} \in S_{2}$, and every triangle of $G_{2}$ has exactly one edge in $S_{2}$. Thus $S:=S_{1} \cup S_{2}$ is a set of edges of $G$ such that $e \in S$, and every triangle of $G$ has exactly one edge in $S$.

By taking as a flip set those edges in the set $S$ from Lemma 3.4 that are in some separating triangle, Lemma 3.2 implies that every triangulation with at least six vertices has a simultaneous flip into a 4-connected triangulation. However, due to the presence of separating triangles, it is not obvious how to implement Lemma 3.4 in linear time. In what follows we show how to do this.

First we outline a few properties of separating triangles. Let $T$ be a separating triangle of a triangulation $G$. Removing the vertices of $T$ from $G$ produces two components, an inner component (containing no vertex on the outerface) and an outer component. Denote by $\operatorname{int}(T)$ and $\operatorname{ext}(T)$ the sets of vertices of the inner and outer components. Define a containment relation, denoted by $\preceq$, on the set of separating triangles of $G$ as follows. For two separating triangles $T_{1}$ and $T_{2}$ of $G$, let $T_{1} \preceq T_{2}$ whenever $\operatorname{int}\left(T_{1}\right) \subseteq \operatorname{int}\left(T_{2}\right)$. Clearly $\preceq$ is a partial order.

We first show how to compute a linear extension $R$ of $\preceq$ in linear time. We then show how to use $R$ to compute the set $S$ in Lemma 3.4 in linear time. The canonical ordering of de Fraysseix et al. [9] will be a useful tool. Let $G$ be a plane triangulation with outerface $(a, b, c)$. A linear ordering of the vertices $\left(v_{1}=a, v_{2}=b, v_{3}, \ldots, v_{n}=c\right)$ is canonical if the following conditions hold for all $3 \leq i \leq n$ :

- the subgraph $G_{i}$ induced by $\left\{v_{1}, v_{2}, \ldots, v_{i}\right\}$ is 2-connected, and the boundary of its outerface is a cycle $C_{i}$ containing the edge $a b$; and

- the vertex $v_{i}$ is in the outerface of $G_{i-1}$, and the neighbours of $v_{i}$ in $G_{i-1}$ form a subinterval of the path $C_{i-1} \backslash\{a b\}$ consisting of at least two vertices (and $v_{3}$ is adjacent to $v_{1}$ and $v_{2}$ ).

de Fraysseix et al. [9] proved that every triangulation has a canonical ordering. Define the level of a separating triangle $T$, denoted by $\ell(T)$, as the largest index of a vertex of $T$ in a given canonical order.

Lemma 3.5. Let $T_{1}$ and $T_{2}$ be separating triangles such that $\ell\left(T_{1}\right)<\ell\left(T_{2}\right)$. Then $T_{1} \preceq T_{2}$ or $\operatorname{int}\left(T_{1}\right) \cap \operatorname{int}\left(T_{2}\right)=\emptyset$.

Proof. Let $T_{1}=(a, b, c)$ and $T_{2}=(x, y, z)$. Suppose on the contrary that $T_{2} \preceq T_{1}$. Then $\operatorname{int}\left(T_{2}\right) \subset \operatorname{int}\left(T_{1}\right)$ since $T_{1}$ and $T_{2}$ are distinct. Without loss of generality, let $c$ be the vertex of $T_{1}$ defining $\ell\left(T_{1}\right)=i$, and let $z$ be the vertex of $T_{2}$ defining $\ell\left(T_{2}\right)=j$. Since $\ell\left(T_{1}\right)=i<\ell\left(T_{2}\right)=j$, $c$ is distinct from $z$. By the canonical ordering, no vertex in $\operatorname{int}\left(T_{1}\right)$ is on the outerface of any $G_{k}$ for $k \geq i$. Since $z$ occurs after $c$ in the canonical ordering, all the vertices adjacent to $z \operatorname{in} \operatorname{int}\left(T_{2}\right)$ are on the outerface of $G_{i}$. This implies that none of these vertices are $\operatorname{in} \operatorname{int}\left(T_{1}\right)$, which is the desired contradiction. 
Lemma 3.6. For an n-vertex plane triangulation $G$, a linear extension $R$ of $\preceq$ can be computed in $\mathcal{O}(n)$ time.

Proof. First note that a canonical ordering can be computed in $\mathcal{O}(n)$ time [9] (also see [6]). Lemma 3.5 implies that if all of the separating triangles of $G$ have different levels, then ordering them by increasing level gives the desired linear extension $R$. What remains is to order the separating triangles at the same level. These triangles share a common vertex $v_{i}$ that defines their level. The neighbours of $v_{i}$ in $G_{i-1}$ form a path $P=\left(p_{1}, p_{2}, \ldots, p_{k}\right)$ on the boundary of the outerface of $G_{i-1}$. Every separating triangle of $G$ at level $i$ consists of $v_{i}$ and two nonconsecutive vertices of $P$. To establish the containment relation between these triangles, we simply need to look at the indices of the vertices of $P$. Let $T_{1}=\left(v_{i}, p_{a}, p_{b}\right)$ and $T_{2}=\left(v_{i}, p_{c}, p_{d}\right)$ be distinct separating triangles with $a<b$ and $c<d$. If $a<b \leq c<d$ or $c<d \leq a<b$ then $\operatorname{int}\left(T_{1}\right) \cap \operatorname{int}\left(T_{2}\right)=\emptyset$ by the canonical ordering. It is impossible for $a<c<b<d$ or $c<a<d<b$ since the graph induced on $P$ is outerplanar and this would violate planarity. If $a \leq c<d \leq b$ then $T_{2} \preceq T_{1}$ and if $c \leq a<b \leq d$ then $T_{1} \preceq T_{2}$. Since we can compute the graph induced by $\left\{p_{1}, p_{2}, \ldots, p_{k}\right\}$ in $\mathcal{O}(k)$ time, all of the separating triangles at level $i$ can be ordered in $\mathcal{O}(k)$ time by performing a breadth-first search on the graph induced on $P$. The result follows since the sum of the degrees of a plane graph is $\mathcal{O}(n)$.

We now turn our attention to computing the set $S$ from Lemma 3.4 in linear time. Denote by FACESet $(G, e)$ the set $S$ from Lemma 3.3, that is, every face of $G$ has exactly one edge in $S$, and if $e$ is specified then $e \in S$.

Algorithm TriangleSet $(G, R)$

Input: triangulation $G$, ordered list of separating triangles $R$ of $G$.

Output: a set $S$ of edges of $G$ such that every triangle of $G$ has exactly one edge in $S$.

1: if $R=\emptyset$ then

2: return FACESET $(G$, unspecified);

3: else

4: $\quad$ let $T$ be the first triangle in $R$;

5: $\quad$ let $S:=$ TriangleSet $(G \backslash \operatorname{int}(T), R \backslash T)$;

6: $\quad$ let $e$ be the edge in $S \cap T$;

7: $\quad$ return $S \cup \operatorname{FACESET}(G \backslash \operatorname{ext}(T), e)$;

8: end if

We now prove the correctness and running time of the algorithm.

Lemma 3.7. For every $n$-vertex triangulation $G$, the algorithm $\operatorname{TRIANGLESET}(G, R)$ returns a set $S$ consisting of exactly one edge in every triangle of $G$. The running time is $\mathcal{O}(n)$.

Proof. We proceed by induction on $|R|$. If $R=\emptyset$ then every triangle in $G$ is a face, and TRIAN$\operatorname{GLESet}(G, R)$ correctly computes $S$ with a call to $\operatorname{FACESet}(G)$. Now assume that $R \neq \emptyset$. Let $T$ be the first triangle in $R$. Then $T$ is an innermost separating triangle of $G$, and $G \backslash \operatorname{ext}(T)$ has no separating triangle. Hence $R \backslash T$ is a linear extension of the containment relation $\preceq$ on the set of separating triangles of $G \backslash \operatorname{int}(T)$. By induction, $S:=\operatorname{TRIANGLESET}(G \backslash \operatorname{int}(T), R \backslash T)$ consists of exactly one edge in every triangle of $G \backslash \operatorname{int}(T)$. Thus there is exactly one edge $e \in S \cap T$. Every triangle in $G \backslash \operatorname{ext}(T)$ is a face. By Lemma 3.3. $\operatorname{FACESET}(G \backslash \operatorname{ext}(T), e)$ consists of exactly one edge in every triangle of $G \backslash \operatorname{ext}(T)$ including $e$. Together with $S$ we have the desired set for $G$. 
The running time is described by the recurrence $X(n)=X(n-|\operatorname{int}(T)|)+\mathcal{O}(|\operatorname{int}(T)|)+\mathcal{O}(1)$, which solves to $\mathcal{O}(n)$.

Note that Algorithm TRIANGLESET can be easily modified to guarantee that a prespecified edge is in $S$.

Theorem 3.8. Every triangulation $G$ with $n \geq 6$ vertices has a simultaneous flip into a 4-connected triangulation that can be computed in $\mathcal{O}(n)$ time.

Proof. By Lemma 3.7 $G$ has a set of edges $S$ such that every separating triangle of $G$ has exactly one edge in $S$ and no triangle of $G$ contains two edges of $S$. By Lemma 3.1, $S$ is flippable. By Lemma $3.2, G\langle S\rangle$ is 4-connected.

We can obtain a stronger result at the expense of a slower algorithm. The following consequence of the 4-colour theorem is essentially a Tait edge-colouring [34].

Lemma 3.9. Every n-vertex planar graph $G$ has an edge 3-colouring that can be computed in $\mathcal{O}\left(n^{2}\right)$ time, such that every triangle is trichromatic.

Proof. Robertson et al. [31] proved that $G$ has a proper vertex 4-colouring that can be computed in $\mathcal{O}\left(n^{2}\right)$ time. Let the colours be $\{1,2,3,4\}$. Colour an edge red if its endpoints are coloured 1 and 2 , or 3 and 4 . Colour an edge blue if its endpoints are coloured 1 and 3 , or 2 and 4 . Colour an edge green if its endpoints are coloured 1 and 4, or 2 and 3. Since the vertices of each triangle $T$ are trichromatic, the edges of $T$ are also trichromatic.

Theorem 3.10. Let $G$ be a triangulation with $n \geq 6$ vertices. Then $G$ has three pairwise disjoint flippable sets of edges $S_{1}, S_{2}, S_{3}$ that can be computed in $\mathcal{O}\left(n^{2}\right)$ time, such that each $G\left\langle S_{i}\right\rangle$ is 4-connected.

Proof. By Lemma 3.9 $G$ has an edge 3-colouring such that every triangle is trichromatic. For any of the three colours, let $S$ be the set of edges receiving that colour and in a separating triangle. By Lemma 3.1 $S$ is flippable. By Lemma 3.2, $G\langle S\rangle$ is 4-connected.

We have the following corollary of Theorems 3.8 and 3.10 since every triangulation on at most five vertices (that is, $K_{3}, K_{4}$ or $K_{5} \backslash e$ ) is Hamiltonian, and every 4-connected triangulation has a Hamiltonian cycle [37] that can be computed in linear time [5].

Theorem 3.11. Every n-vertex triangulation $G$ has a simultaneous flip into a Hamiltonian triangulation that can be computed in $\mathcal{O}(n)$ time. Furthermore, $G$ has three disjoint simultaneous flips that can be computed in $\mathcal{O}\left(n^{2}\right)$ time, such that each transforms $G$ into a Hamiltonian triangulation.

\section{Outerplane Graphs}

A plane graph is outerplane if every vertex lies on the outerface. The other faces are internal. An edge that is not on the boundary of the outerface is internal. Let $G$ be an (edge-)maximal outerplane graph $G$ on $n$ vertices. Every internal face is a triangle, and $G$ has $2 n-3$ edges and $n-2$ internal faces. The dual tree of $G$, denoted by $G^{*}$, is the dual graph of $G$ without a vertex 
corresponding to the outerface. Observe that $G^{*}$ is a tree with $n-2$ vertices and maximum degree at most three.

The notions of diagonal flip and flippable set for triangulations are extended to maximal outerplane graphs in the natural way, except that only internal edges are allowed to be flipped. (It is not clear what it means to flip an edge of the outerface since for $n>3$, the outerface is not a triangle.) A flip in an outerplanar graph corresponds to a certain rotation in the dual tree; see [4, 28, 29, 32]. This section focuses on simulatenous flips in maximal outerplane graphs, which have not previously been studied.

Lemma 4.1. Every internal edge of a maximal outerplane graph is flippable.

Proof. Suppose that an internal edge $v w$ is not filppable. Then $v w$ is blocked by some edge $p q$. Thus $\{v, w, p, q\}$ induce $K_{4}$. This is a contradiction since no outerplane graph contains $K_{4}$.

Lemma 4.2. A set $S$ of internal edges in a maximal outerplane graph $G$ is flippable if and only if the corresponding dual edges $S^{*}$ form a matching in $G^{*}$.

Proof. For $S$ to be flippable it is necessary that there are no two consecutive edges in $S$. This is equivalent to the condition that $S^{*}$ is a matching of $G^{*}$. By Lemma 4.1, every edge in $S$ is flippable. As in Lemma 2.1 the only obstruction to $S$ being flippable is a bad pair of edges, which cannot occur since a bad pair of edges contains a subdivision of $K_{4}$.

Theorem 4.3. Every n-vertex maximal outerplane graph $G$ has a flippable set of at least $\frac{1}{3}(n-3)$ edges. Moreover, for infinitely many $n$, there is an $n$-vertex maximal outerplane graph in which every flippable set has at most $\frac{1}{3}(n-3)$ edges.

Proof. First we prove the lower bound. Since $G^{*}$ is a tree with maximum degree at most three, $G^{*}$ has a proper edge 3 -colouring (by an easy inductive argument). Now $G^{*}$ has $n-3$ edges. Thus the largest colour class is a matching of at least $\frac{1}{3}(n-3)$ edges, which by Lemma 4.2 corresponds to a flippable set of at least $\frac{1}{3}(n-3)$ edges in $G$.

Now we prove the upper bound. By Lemma4.2 it suffices to construct trees $T$ with maximum degree three, in which the maximum cardinality of a matching equals one third the number of edges. We can then take the maximal outerplane graph $G$ for which $G^{*}=T$. Let $T$ be a tree rooted at a vertex $r$ such that every non-leaf vertex has degree three, and the distance between every leaf vertex and $r$ is odd. Obviously there are infinitely many such trees. Let $K$ be the set of vertices at even distance from $r$. Then $K$ is a vertex cover of $T$ (that is, every edge of $T$ is incident to a vertex in $K$ ). Since no edge of $T$ has both its endpoints in $K$, and every vertex in $K$ has degree three, $|K|$ equals one third the number of edges. Since $T$ has maximum degree three, $K$ is a minimum vertex cover. König [19] proved that the maximum cardinality of a matching in a bipartite graph equals the minimum cardinality of a vertex cover. Thus the maximum cardinality of a matching equals one third the number of edges, as desired.

The following is the main result of this section. In the remainder of this paper all logarithms have base 2 , and $c_{1}$ is the constant $2 / \log \frac{6}{5}(\approx 7.6)$.

Theorem 4.4. Let $G_{1}$ and $G_{2}$ be (unlabelled) maximal outerplane graphs on $n$ vertices. There is a sequence of at most $4 c_{1} \log n$ simultaneous flips to transform $G_{1}$ into $G_{2}$.

Theorem 4.4 is implied by the following lemma. 
Lemma 4.5. For every maximal outerplane graph $G$ on $n$ vertices, there is a sequence of at most $2 c_{1} \log n$ simultaneous flips to transform $G$ into a maximal outerplane graph that has a dominant vertex.

Proof of Theorem 4.4 assuming Lemma4.5 Observe that two $n$-vertex maximal outerplane graphs each with a dominant vertex are isomorphic. Let $D_{n}$ denote the $n$-vertex maximal outerplane graph with a dominant vertex. To transform $G_{1}$ into $G_{2}$, first transform $G_{1}$ into $D_{n}$, and then apply the flips to transform $G_{2}$ into $D_{n}$ in reverse order.

The proof of Lemma 4.5 proceeds in two parts. In Lemma 4.6 we reduce the diameter of the dual tree to $c_{1} \log n$ using at most $c_{1} \log n$ simultaneous flips. Then in Lemma 4.7 a dominant vertex is introduced using at most a further $c_{1} \log n$ simultaneous flips.

Lemma 4.6. Let $G$ be a maximal outerplane graph on $n$ vertices. Then $G$ can be transformed by a sequence of at most $c_{1} \log n$ simultaneous flips into a maximal outerplane graph $X$ such that the diameter of the dual tree $X^{*}$ is at most $c_{1} \log n$.

Proof. We proceed by induction on $n$. The result holds trivially for $n=3$. Assume the lemma holds for graphs with less than $n$ vertices, and let $G$ be a maximal outerplane graph on $n$ vertices. By a theorem of Bose et al. [2], $G$ has an independent set $I$ of at least $\frac{n}{6}$ vertices, and $\operatorname{deg}_{G}(v) \leq 4$ for every vertex $v \in I$. Obviously $\operatorname{deg}_{G}(v) \geq 2$. For $d \in\{2,3,4\}$, let $I_{d}:=\{v \in I$ : $\left.\operatorname{deg}_{G}(v)=d\right\}$.

For every vertex $v \in I_{3} \cup I_{4}$, add one internal edge incident to $v$ to a set $S$. Since $I$ is independent, $|S|=\left|I_{3}\right|+\left|I_{4}\right|$. Suppose on the contrary that there are two consecutive edges $x u, x v \in S$. Then $x \notin I_{3} \cup I_{4}$, which implies that $u, v \in I_{3} \cup I_{4}$. Since every internal face of $G$ is a triangle, $u v$ is an edge of $G$, which contradicts the independence of $I$. Thus no two edges in $S$ are consecutive. By Lemma 4.2 $S$ is flippable in $G$. Let $G^{\prime}:=G\langle S\rangle$. Every vertex $v \in I_{2} \cup I_{3}$ has $\operatorname{deg}_{G^{\prime}}(v)=2$, and every vertex $v \in I_{4}$ has $\operatorname{deg}_{G^{\prime}}(v)=3$.

Since $I_{4}$ is an independent set of $G$, and any edge in $G^{\prime}$ that is incident to a vertex in $I_{4}$ is also in $G, I_{4}$ is an independent set of $G^{\prime}$. Let $S^{\prime}$ be the set of internal edges of $G^{\prime}$ incident to a vertex in $I_{4}$. Thus $\left|S^{\prime}\right|=\left|I_{4}\right|$, and by the same argument used for $S$, no two edges in $S^{\prime}$ are consecutive in $G^{\prime}$. By Lemma 4.2, $S^{\prime}$ is flippable in $G^{\prime}$. Let $G^{\prime \prime}:=G^{\prime}\left\langle S^{\prime}\right\rangle$. Every vertex $v \in I$ has $\operatorname{deg}_{G^{\prime \prime}}(v)=2$.

Thus $G$ can be transformed by two simultaneous flips into a maximal outerplane graph $G^{\prime \prime}$ containing at least $\frac{n}{6}$ vertices of degree two. Let $G^{\prime \prime \prime}$ be the maximal outerplane graph obtained from $G^{\prime \prime}$ by deleting the vertices of degree two. Then $G^{\prime \prime \prime}$ has at most $\frac{5}{6} n$ vertices. By induction, $G^{\prime \prime \prime}$ can be transformed by a sequence of at most $c_{1} \log \frac{5}{6} n$ simultaneous flips into a maximal outerplane graph $X$ such that the diameter of $X^{*}$ is at most $c_{1} \log \frac{5}{6} n$.

Consider a vertex $v \in I$. Since $\operatorname{deg}_{G^{\prime \prime}}(v)=2$, there is one internal face incident to $v$ in $G^{\prime \prime}$, which corresponds to a leaf in $G^{\prime \prime *}$. Thus the dual tree $X^{*}$ is obtained by adding leaves to the dual tree $G^{\prime \prime *}$. Hence the diameter of $X^{*}$ is at most the diameter of $G^{\prime \prime *}$ plus 2, which is $2+c_{1} \log \frac{5}{6} n=c_{1} \log n$. We have used two simultaneous flips, $S$ and $S^{\prime}$, to transform $G$ into $G^{\prime \prime}$, and then $c_{1} \log \frac{5}{6} n$ simultaneous flips to transform $G^{\prime \prime}$ into $X$. The total number of flips is $2+c_{1} \log \frac{5}{6} n=c_{1} \log n$.

Lemma 4.7. Let $G$ be a maximal outerplane graph on $n$ vertices. Suppose that $G^{*}$ has diameter $k$. Let $v$ be a fixed vertex of $G$. Then $G$ can be transformed by at most $k$ simultaneous flips into a maximal outerplane graph $X$ in which $v$ is dominant. 
Proof. As illustrated in Figure 6, let $P$ be the set of internal faces incident with $v$ in $G$. In the dual tree $G^{*}$, the corresponding vertices of $P$ form a path $P^{*}$. Define the distance of each vertex $x$ in $G^{*}$ as the minimum number of edges in a path from $x$ to a vertex in $P^{*}$. Since the diameter of $G^{*}$ is $k$, every vertex in $G^{*}$ has distance at most $k$. No two vertices in $G^{*}$ both with distance one are adjacent, as otherwise $G^{*}$ would contain a cycle. Each vertex of $P^{*}$ is adjacent to at most one vertex at distance one, since $G^{*}$ has maximum degree at most three, and the endpoints of $P^{*}$ correspond to faces with an edge on the outerface of $G$. Let $S^{*}$ be the set of edges of $G^{*}$ incident to $P^{*}$ but not in $P^{*}$. Then $S^{*}$ is a matching between the vertices at distance one and the vertices of $P^{*}$, such that all vertices at distance one are matched. Let $S$ be the set of edges of $G$ corresponding to $S^{*}$ under duality. Note that $S$ is the set of internal edges that are seen by $v$. By Lemma 4.2 $S$ is a flippable set of edges of $G$. Let $G^{\prime}:=G\langle S\rangle$. In $G^{\prime}$, the distance of each vertex not adjacent to $P^{*}$ is reduced by one. Thus, by induction, at most $k$ simultaneous flips are required to reduce the distance of every vertex to zero, in which case $v$ is adjacent to every other vertex.

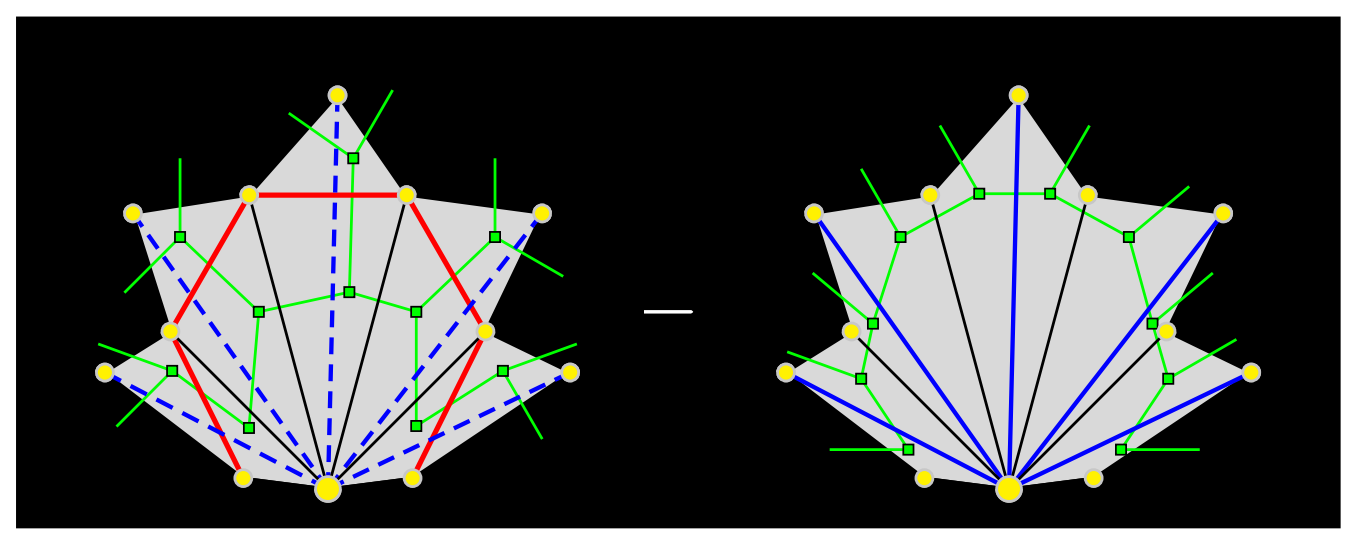

Figure 6: Making $v$ a dominant vertex in Lemma 4.7 the vertices of the dual tree are drawn as squares.

Clearly Lemma 4.5 is implied by Lemmata 4.6 and 4.7 (with $k=c_{1} \log n$ ).

\section{Simultaneous Flips Between Given Triangulations}

In this section we prove the following theorem, which is an analogue of Theorem 4.4 for outerplane graphs. Throughout, $c_{1}$ is the constant $2 / \log \frac{6}{5}(\approx 7.6)$ from Section 4 and $c_{2}$ is the constant $2 / \log \frac{54}{53}(\approx 74.2)$.

Theorem 5.1. Let $G_{1}$ and $G_{2}$ be (unlabelled) triangulations on $n$ vertices. There is a sequence of at most $2+4\left(c_{1}+c_{2}\right) \log n$ simultaneous flips to transform $G_{1}$ into $G_{2}$.

Theorem 5.1 is implied by the following lemma using the approach of Wagner described in Section 1

Lemma 5.2. For every $n$-vertex triangulation $G$, there is a sequence of at most $1+2\left(c_{1}+c_{2}\right) \log n$ simultaneous flips to transform $G$ into the standard triangulation $\Delta_{n}$. 
To prove Lemma 5.2 we first apply Theorem 3.11 to obtain a Hamiltonian triangulation with one simultaneous flip. Thus it suffices to prove that a Hamiltonian triangulation can be transformed into $\Delta_{n}$. A Hamiltonian cycle $H$ of a triangulation $G$ naturally divides $G$ into two maximal outerplane subgraphs: an 'inner' subgraph consisting of $H$ and the edges inside $H$, and an 'outer' subgraph consisting of $H$ and the edges outside of $H$. (Note that Mori et al. [20] used a similar approach for individual flips.) At this point, it is tempting to apply Lemma 4.5twice, once on the inner subgraph to obtain one dominant vertex, and then on the outer subgraph to obtain a second dominant vertex, thus reaching the standard triangulation. However, Lemma 4.5 cannot be applied directly since we need to take into consideration the interaction between these two outerplane subgraphs. The main problem is that an internal edge in the inner subgraph may be blocked by an edge in the outer subgraph. The bulk of this section is dedicated to solving this impasse.

First some definitions. A chord of a cycle $C$ in a triangulation $G$ is an edge of $G$ that is not in $C$ and whose endpoints are both in $C$. A chord $e$ of $C$ is classified as internal or external depending on whether $e$ is contained in the interior or exterior of $C$ (with respect to the outerface of $G$ ). For the inductive step in Lemma 5.5 below to work we need to consider a more general type of cycle than a Hamiltonian cycle. A cycle $C$ of a triangulation $G$ is empty if the interior of $C$ contains no vertices of $G$. Obviously a Hamiltonian cycle is always empty. For an empty cycle $C$ of a triangulation $G$, let $G\{C\}$ denote the subgraph of $G$ whose vertices are the vertices of $C$, and whose edges are the edges of $C$ along with the internal chords of $C$. Then $G\{C\}$ is a maximal outerplane graph, and the boundary of the outerface of $G\{C\}$ is $C$.

Lemma 5.3. Let $C$ be an empty cycle of a triangulation $G \neq K_{4}$. Let $v w$ be an internal chord of $C$ that is blocked by some edge $p q$. Then $p q$ is an external chord of $C$ that is flippable in $G$.

Proof. By Lemma 2.4 $p q$ is a flippable edge of $G$. Since $C$ is empty, $p$ and $q$ are vertices of $C$. Now $p q$ is not internal, as otherwise $\{p, q, v, w\}$ would induce $K_{4}$ in the outerplane graph $G\{C\}$. Thus $p q$ is external.

Lemma 5.4. Let $C$ be an empty cycle of a triangulation $G$. Let $S$ be a set of internal chords of $C$, no two of which are consecutive. Then there is a flippable set $T$ of edges in $G$ such that:

(a) $T \cap C=\emptyset$,

(b) $|S \cap T| \geq \frac{1}{3}|S|$, and

(c) every edge in $T \backslash S$ is an external chord of $C$ and $|T \backslash S| \leq|S \cap T|$.

Proof. Let $S^{\prime}$ be the set of edges in $S$ that are individually flippable in $G$. Let $S^{\prime \prime}:=S \backslash S^{\prime}$. By Lemma 5.3. there is an external chord that blocks each edge $e \in S^{\prime \prime}$. Distinct edges $e_{1}, e_{2} \in S^{\prime \prime}$ are blocked by distinct external chords, as otherwise $e_{1}$ and $e_{2}$ would be a bad pair, and the outerplane graph $G\{C\}$ would contain a subdivision of $K_{4}$. Let $B$ be this set of blocking external chords. Thus $|B|=\left|S^{\prime \prime}\right|$. By Lemma 3.9 $B$ can be 3-coloured such that no two monochromatic edges in $B$ are consecutive in $G$. (Note that since $B$ forms an outerplane subgraph of $G$, this 3 -colouring can be computed in $\mathcal{O}(n)$ time without using the 4-colour theorem.) Let $P$ be the largest set of monochromatic edges in $B$. Then $|P| \geq \frac{1}{3}|B|$. Let $Q$ be the set of edges in $S^{\prime \prime}$ that are blocked by edges in $P$. Then $|Q|=|P|$. Let $T:=S^{\prime} \cup P \cup Q$. Observe that $T \cap C=\emptyset$. This proves (a).

To prove that $T$ is flippable in $G$, we verify each of the conditions of Lemma 2.1 $T$ consists of internal chords $S^{\prime} \cup Q$, and external chords $P$. Since $S^{\prime} \cup Q \subseteq S$, no two internal chords in 
$T$ are consecutive. By the construction of $P$, no two external chords in $T$ are consecutive. Since the internal chords and external chords are separated by $C$, no two edges in $T$ are consecutive. Thus condition (1) of Lemma 2.1 is satisfied.

As in Lemma 4.2, there is no bad pair among the internal chords as otherwise $G\{C\}$ would contain a subdivision of $K_{4}$. Similarly there is no bad pair among the external chords. Suppose there is a bad pair of edges in $T$, one an internal chord $x y$ and the other an external chord $v w$. Then both $v w$ and $x y$ are seen by some pair of vertices $p$ and $q$. Since $v w \in P \subseteq B$, $v w$ blocks some internal chord $a b \in S^{\prime \prime}$. By Lemma 2.5, $a b$ and $x y$ are consecutive, which is a contradiction since both edges are in $S$. Thus there is no bad pair in $T$, and condition (2) of Lemma 2.1 is satisfied.

Each edge in $P$ blocks some other edge, and is thus individually flippable by Lemma 2.4 By definition, all the edges in $S^{\prime}$ are individually flippable in $G$. While each edge in $Q$ is not individually flippable, the corresponding blocking edge is in $P \subseteq T$. Thus condition (3) of Lemma 2.1 is satisfied. Therefore $T$ is flippable in $G$.

Now $T \cap S=S^{\prime} \cup Q$. Since $S^{\prime} \cap Q=\emptyset$, we have $|T \cap S|=\left|S^{\prime}\right|+|Q|=\left|S^{\prime}\right|+|P| \geq$ $\left|S^{\prime}\right|+\frac{1}{3}|B| \geq \frac{1}{3}\left|S^{\prime}\right|+\frac{1}{3}\left|S^{\prime \prime}\right|=\frac{1}{3}|S|$. This proves (b). Now $T \backslash S=P$, all of whose elements are external chords. Since $|S \cap T|=\left|S^{\prime}\right|+|P|$, we have $|P| \leq|S \cap T|$. Since $T \backslash S=P$, we have $|T \backslash S| \leq|S \cap T|$. This proves (c).

The following result extends Lemma 4.6 for outerplane graphs to the case of triangulations.

Lemma 5.5. Let $H$ be a Hamiltonian cycle of a triangulation $G$ with $n$ vertices. Then $G$ can be transformed by a sequence of at most $c_{2} \log n$ simultaneous flips into a triangulation $X$ in which $H$ is a Hamiltonian cycle and the diameter of $X\{H\}^{*}$ is at most $c_{2} \log n$.

Proof. We proceed by induction on $n$ with the following stronger hypothesis:

"Let $G$ be a triangulation, and let $C$ be an empty cycle of $G$ with $n$ vertices. ( $G$ may have more than $n$ vertices.) Then $G$ can be transformed by a sequence of at most $c_{2} \log n$ simultaneous flips into a triangulation $X$ in which $C$ is an empty cycle and the diameter of $X\{C\}$ is at most $c_{2} \log n$. Moreover, every edge of $G$ that is incident to a vertex not in $C$ remains in $X$."

The lemma immediately follows since any Hamiltonian cycle is empty. The hypothesis holds trivially for $n=3$. Assume the hypothesis holds for all triangulations with less than $n$ vertices. Let $G$ be a triangulation, and let $C$ be an empty cycle of $G$ with $n$ vertices.

By a theorem of Bose et al. [2], the outerplane graph $G\{C\}$ has an independent set $I$ of at least $\frac{n}{6}$ vertices, and $\operatorname{deg}_{G\{C\}}(v) \leq 4$ for every vertex $v \in I$. Obviously $\operatorname{deg}_{G\{C\}}(v) \geq 2$. For $d \in\{2,3,4\}$, let $I_{d}:=\left\{v \in I: \operatorname{deg}_{G\{C\}}(v)=d\right\}$.

For every vertex $v \in I_{3} \cup I_{4}$, add one internal chord of $C$ that is incident to $v$ to a set $S$. Since $I$ is independent, $|S|=\left|I_{3}\right|+\left|I_{4}\right|$. Suppose on the contrary that there are two consecutive edges $x u$ and $x v$ in $S$. Then $x \notin I_{3} \cup I_{4}$, which implies that $u, v \in I_{3} \cup I_{4}$. Since every face of $G$ is a triangle, $u v$ is an edge, which contradicts the independence of $I$. Thus no two edges in $S$ are consecutive. By Lemma[5.4 there is a flippable set of edges $T$ in $G$, such that $T \cap C=\emptyset$ and $|S \cap T| \geq \frac{1}{3}|S|=\frac{1}{3}\left(\left|I_{3}\right|+\left|I_{4}\right|\right)$. Moreover, every edge in $T \backslash S$ is an external chord of $C$ in $G$. For $d \in\{3,4\}$, let $I_{d}^{\prime}$ be the set of vertices in $I_{d}$ incident to an edge in $S \cap T$. Thus $\left|I_{3}^{\prime}\right|+\left|I_{4}^{\prime}\right| \geq \frac{1}{3}\left(\left|I_{3}\right|+\left|I_{4}\right|\right)$.

Let $G^{\prime}:=G\langle T\rangle$. Since $T \cap C=\emptyset, C$ is an empty cycle of $G^{\prime}$. Every vertex $v \in I_{2} \cup I_{3}^{\prime}$ has $\operatorname{deg}_{G^{\prime}\{C\}}(v)=2$. Every vertex $v \in I_{4}^{\prime}$ has $\operatorname{deg}_{G^{\prime}\{C\}}(v)=3$. 
An edge in $G^{\prime}\{C\}$ that is incident to a vertex in $I_{4}^{\prime}$ is also in $G\{C\}$. Since $I_{4}^{\prime}$ is an independent set of $G\{C\}$, it is also an independent set of $G^{\prime}\{C\}$. Let $S^{\prime}$ be the set of internal chords of $C$ in $G^{\prime}$ that are incident to a vertex in $I_{4}^{\prime}$. Thus $\left|S^{\prime}\right|=\left|I_{4}^{\prime}\right|$, and by the same argument used for $S$, no two edges in $S^{\prime}$ are consecutive in $G^{\prime}$. By Lemma 5.4 there is a flippable set of edges $T^{\prime}$ in $G^{\prime}$, such that $T^{\prime} \cap C=\emptyset$ and $\left|S^{\prime} \cap T^{\prime}\right| \geq \frac{1}{3}\left|S^{\prime}\right|=\frac{1}{3}\left|I_{4}^{\prime}\right|$. Moreover, every edge in $T^{\prime} \backslash S^{\prime}$ is an external chord of $C$ in $G^{\prime}$. Let $I_{4}^{\prime \prime}$ be the set of vertices in $I_{4}^{\prime}$ incident to an edge in $S^{\prime} \cap T^{\prime}$. Thus $\left|I_{4}^{\prime \prime}\right| \geq \frac{1}{3}\left|I_{4}^{\prime}\right|$.

Let $G^{\prime \prime}:=G^{\prime}\left\langle T^{\prime}\right\rangle$. Since $T^{\prime} \cap C=\emptyset, C$ is an empty cycle of $G^{\prime \prime}$. Every vertex $v \in I_{2} \cup I_{3}^{\prime} \cup I_{4}^{\prime \prime}$ has $\operatorname{deg}_{G^{\prime \prime}\{C\}}(v)=2$. Now $\left|I_{2} \cup I_{3}^{\prime} \cup I_{4}^{\prime \prime}\right| \geq\left|I_{2}\right|+\left|I_{3}^{\prime}\right|+\frac{1}{3}\left|I_{4}^{\prime}\right| \geq\left|I_{2}\right|+\frac{1}{3}\left(\left|I_{3}^{\prime}\right|+\left|I_{4}^{\prime}\right|\right) \geq\left|I_{2}\right|+\frac{1}{9}\left(\left|I_{3}\right|+\left|I_{4}\right|\right) \geq$ $\frac{1}{9}\left(\left|I_{2}\right|+\left|I_{3}\right|+\left|I_{4}\right|\right)=\frac{1}{9}|I| \geq \frac{n}{54}$.

In summary, $G$ can be transformed by two simultaneous flips into a triangulation $G^{\prime \prime}$ in which $C$ is an empty cycle, and $G^{\prime \prime}\{C\}$ has an independent set $L\left(=I_{2} \cup I_{3}^{\prime} \cup I_{4}^{\prime \prime}\right)$ such that $|L| \geq \frac{n}{54}$ and $\operatorname{deg}_{G^{\prime \prime}\{C\}}(v)=2$ for every vertex $v \in L$. Consider a vertex $v \in L$. Say $(u, v, w)$ is the 2-edge path in $C$. Since $L$ is independent, $u \notin L$ and $w \notin L$. Since $\operatorname{deg}_{G^{\prime \prime}\{C\}}(v)=2$, $u w$ is an internal chord of $C$ in $G^{\prime \prime}$. Let $D$ be the cycle of $G$ obtained by replacing the the path $(u, v, w)$ in $C$ by the edge $u w$ (for all $v \in L$ ). Thus $D$ is an empty cycle of $G^{\prime \prime}$, and $|D|=n-|L| \leq \frac{53}{54} n$. By induction applied to $D$ and $G^{\prime \prime}, G^{\prime \prime}$ can be transformed by a sequence of at most $c_{2} \log \frac{53}{54} n$ simultaneous flips into a triangulation $X$ in which $D$ is an empty cycle and the diameter of $X\{D\}^{*}$ is at most $c_{2} \log \frac{53}{54} n$. Moreover, every edge of $G^{\prime \prime}$ that is incident to a vertex not in $D$ remains in $X$.

Consider a vertex $v \in L$. Say $(u, v, w)$ is the 2-edge path in $C$. Since $v$ is not in $D$, the edges $u v$ and $v w$ of $G$ are in $X$. Thus $C$ is an empty cycle of $X$. Since $u w$ is an edge of $D$, uvw is a face of $X$. The vertex in the dual tree $X\{C\}^{*}$ that corresponds to uvw is a leaf in $X\{C\}^{*}$. Thus $X\{C\}^{*}$ is obtained by adding leaves to the dual tree $X\{D\}^{*}$. Hence the diameter of $X\{C\}^{*}$ is at most the diameter of $X\{D\}^{*}$ plus 2, which is at most $2+c_{2} \log \frac{53}{54} n=c_{2} \log n$. We have used two simultaneous flips, $T$ and $T^{\prime}$, to transform $G$ into $G^{\prime \prime}$, and then at most $c_{2} \log \frac{53}{54} n$ simultaneous flips to transform $G^{\prime \prime}$ into $X$. The total number of flips is at most $2+c_{2} \log \frac{53}{54} n=c_{2} \log n$. Since every edge in $T$ is a chord of $C$ in $G$, and every edge in $T^{\prime}$ is a chord of $C$ in $G^{\prime}$, every edge of $G$ that is incident to a vertex not in $C$ remains in $X$.

The following result is analogous to Lemma 4.7 for outerplane graphs. The key difference is that the choice of vertex $v$ is no longer arbitrary.

Lemma 5.6. Let $H$ be a Hamiltonian cycle of a triangulation $G$. Suppose that $G\{H\}^{*}$ has diameter $k$. Let $v$ be a vertex of $G$ not incident to any external chords of $H$ in $G$. Then $G$ can be transformed by at most $k$ simultaneous flips into a triangulation $X$ in which $H$ is a Hamiltonian cycle of $X$ and $v$ is dominant. Moreover, every edge incident to $v$ is in $X\{H\}$.

Proof. First note that there is such a vertex $v$ since the subgraph of $G$ consisting of $H$ and the external chords of $H$ is maximal outerplane, and thus has a vertex of degree two. Let $P$ be the set of internal faces incident with $v$ in $G$. In the dual tree $G\{H\}^{*}$, the corresponding vertices of $P$ form a path $P^{*}$. Define the distance of each vertex $x$ in $G\{H\}^{*}$ as the minimum number of edges in a path from $x$ to a vertex in $P^{*}$. Since the diameter of $G\{H\}^{*}$ is $k$, every vertex in $G^{*}$ has distance at most $k$. No two vertices in $G\{H\}^{*}$ both with distance one are adjacent, as otherwise $G\{H\}^{*}$ would contain a cycle. Each vertex of $P^{*}$ is adjacent to at most one vertex at distance one, since $G\{H\}^{*}$ has maximum degree at most three, and the endpoints of $P^{*}$ correspond to faces with an edge on the outerface of $G\{H\}$. Let $S^{*}$ be the set of edges of $G\{H\}^{*}$ incident to $P^{*}$ but not in $P^{*}$. Then $S^{*}$ is a matching between the vertices at distance 
one and the vertices of $P^{*}$, such that all vertices at distance one are matched. Let $S$ be the set of edges of $G\{H\}$ corresponding to $S^{*}$ under duality. Consider an edge $x y \in S$. Then $x y$ is seen by $v$ and some other vertex $w$. If $x y$ is not flippable, then by Lemma[5.3, $v w$ is an external chord of $H$ in $G$. Thus $x y$ is flippable, since by construction, $v$ is not incident to any external chords of $H$ in $G$. Hence $S$ is a set of individually flippable edges. No two edges in $S$ are consecutive, since every internal face of $G\{H\}$ is a triangle. No two edges in $G\{H\}$ form a bad pair since $G\{H\}$ is outerplane. By Lemma 2.1 $S$ is flippable in $G$. Let $G^{\prime}:=G\langle S\rangle$. Observe that $S \cap H=\emptyset$. Thus $H$ is a Hamiltonian cycle of $G^{\prime}$. In $G^{\prime}\{H\}$, the distance of each vertex not adjacent to $P^{*}$ is reduced by one. Thus, by induction, at most $k$ simultaneous flips are required to reduce the distance of every vertex to zero, in which case $v$ is adjacent to every other vertex of $G$, and every edge incident to $v$ is in $G\{H\}$.

Lemmata 5.5 and 5.6 imply:

Lemma 5.7. Let $H$ be a Hamiltonian cycle of a triangulation $G$. Then $G$ can be transformed by at most $2 c_{2} \log n$ simultaneous flips into a triangulation $X$ in which $H$ is a Hamiltonian cycle of $X$, and there is a vertex $v$ adjacent to every other vertex, and every edge incident to $v$ is in $X\{H\}$.

We are now half way to transforming a given triangulation into the standard triangulation. The second half is somewhat easier.

Lemma 5.8. Let $G$ be an $n$-vertex triangulation with a dominant vertex $v$. Then there is a sequence of at most $2 c_{1} \log (n-1)$ simultaneous flips to transform $G$ into the standard triangulation on $n$ vertices.

Proof. Observe that $G \backslash v$ is a maximal outerplane graph, in which the vertices are ordered on the outerface according to the cyclic order of the neighbours of $v$. Let $C$ be the cycle bounding the outerface of $G \backslash v$. By Lemma 4.5 there is a sequence of at most $2 c_{1} \log (n-1)$ simultaneous flips to transform $G \backslash v$ into a maximal outerplane graph with a dominant vertex. Each of these flips is valid in $G$ since $C$ has no internal chords (cf. Lemma 5.4). We obtain the standard triangulation.

Observe that Lemmata 5.7 and 5.8 together prove Lemma 5.2 which in turn proves Theorem 5.1 Although the $\mathcal{O}(\log n)$ simultaneous flips in Theorem 5.1 may each involve a linear number of edges, the total number of flipped edges is linear.

Theorem 5.9. Let $G_{1}$ and $G_{2}$ be triangulations on $n$ vertices. There is a sequence of $\mathcal{O}(\log n)$ simultaneous flips to transform $G_{1}$ into $G_{2}$, and $\mathcal{O}(n)$ edges are flipped in total.

Proof. It suffices to prove that there are $\mathcal{O}(n)$ flips in Lemmata 5.5 and 5.6 since at most $n$ edges are flipped to make the graph Hamiltonian, and there are constant times as many flips in Theorem 5.1 as there are in Lemmata 5.6 and 5.5 In Lemma 5.6 each flipped edge becomes incident to $v$, and then remains incident to $v$. Thus the number of flipped edges is at most $n-1$. In Lemma 5.5 $\mathcal{O}(n)$ edges are flipped to obtain a triangulation on at most $\frac{53}{54} n$ vertices. Therefore, the number of flipped edges $F(n)$ satisfies the recurrence $F(n)=F\left(\frac{53}{54} n\right)+\mathcal{O}(n)$, which solves to $\mathcal{O}(n)$. 


\section{Large Simultaneous Flips}

In this section we prove bounds on the size of a maximum simultaneous flip in a triangulation. Let $\operatorname{msf}(G)$ denote the maximum cardinality of a flippable set of edges in a triangulation $G$. In related work, Gao et al. [11] proved that every triangulation has at least $n-2$ (individually) flippable edges, and every triangulation with minimum degree four has at least $2 n+3$ (individually) flippable edges. Galtier et al. [10] proved that every geometric triangulation has a set of at least $\frac{1}{6}(n-4)$ simultaneously flippable edges. The following is the main result of this section.

Theorem 6.1. For every triangulation $G$ with $n \geq 4$ vertices, $\operatorname{msf}(G) \geq \frac{1}{3}(n-2)$.

Assume there is a counterexample to Theorem 6.1 that is, a triangulation $G$ with $n \geq 4$ vertices and $\operatorname{msf}(G)<\frac{1}{3}(n-2)$. A counterexample with the minimum number of vertices is a minimum counterexample.

Lemma 6.2. A counterexample has $n \geq 7$ vertices.

Proof. If $n=4$ then $G=K_{4}$, which has a flippable set of $2>\frac{1}{3}(4-2)$ edges, as illustrated in Figure Z(a). If $n=5$ then $G=K_{5} \backslash e$, which has a flippable set of $2>\frac{1}{3}(5-2)$ edges.

(a)

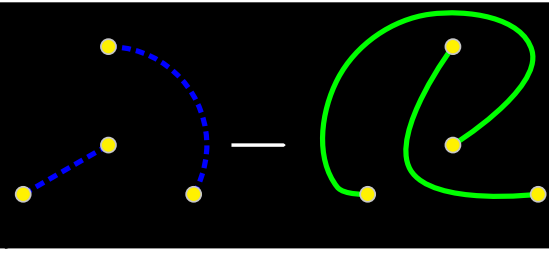

(b)

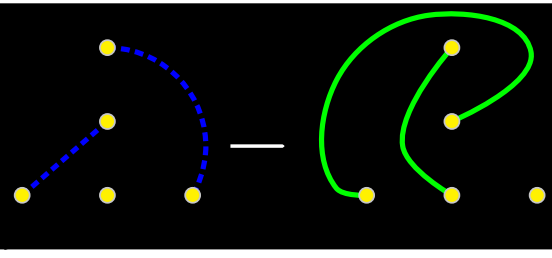

Figure 7: 2-edge flip in (a) $K_{4}$ and (b) $K_{5} \backslash e$.

If $n=6$ then $G$ is the octahedron illustrated in Figure 8 (a), or $G$ is the triangulation illustrated in Figure 8 (b). In both cases there is a flippable set of $3>\frac{1}{3}(6-2)$ edges.

(a)

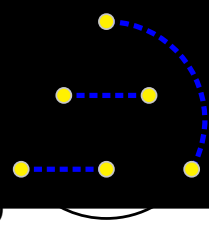

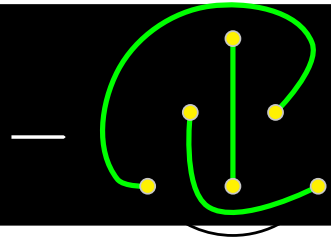

(b)

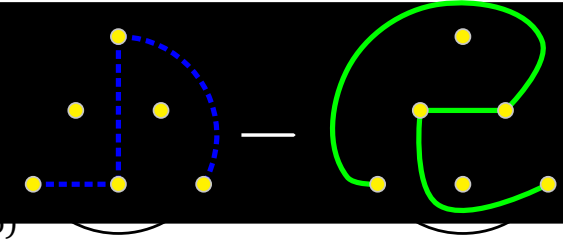

Figure 8: 3-edge flip in (a) the octahedron and (b) the other 6-vertex triangulation.

Lemma 6.3. A minimum counterexample has no edge vw with $\operatorname{deg}(v)=3$ and $\operatorname{deg}(w)=4$.

Proof. Let $G$ be a minimum counterexample with $n$ vertices. Suppose that $G$ has an edge $v w$ with $\operatorname{deg}(v)=3$ and $\operatorname{deg}(w)=4$. Then the neighbours of $v$ and $w$ form a triangle $(x, y, z)$ with $v$ adjacent to $x$ and $y$, and $w$ adjacent to $x, y$ and $z$. Let $G^{\prime}:=(G \backslash v) \backslash w$. Then $G^{\prime}$ is a triangulation with $n-2$ vertices in which $(x, y, z)$ is a face. Since $G$ is minimum, $G^{\prime}$ is not a counterexample. Thus $G^{\prime}$ has a flippable set $S^{\prime}$ of at least $\frac{1}{3}(n-4)$ edges. At most one of $\{x y, x z, y z\}$ is in $S^{\prime}$. 
If $x z \in S^{\prime}$, then let $S:=S^{\prime} \cup\{y w\}$. Otherwise let $S:=S^{\prime} \cup\{x w\}$. It is trivial to check that $S$ is a flippable set of $G$. Moreover, $|S|=\left|S^{\prime}\right|+1 \geq \frac{1}{3}(n-4)+1>\frac{1}{3}(n-2)$. Thus $G$ is not a counterexample.

Lemma 6.4. A minimum counterexample has no edge vw with $\operatorname{deg}(v)=4$ and $\operatorname{deg}(w)=4$.

Proof. Let $G$ be a minimum counterexample with $n$ vertices. Suppose that $G$ has an edge $v w$ with $\operatorname{deg}(v)=4$ and $\operatorname{deg}(w)=4$. Let $b$ and $d$ be the vertices that see $v w$. Then $b \neq d$, as otherwise $G=K_{3}$. Let $a$ be the other neighbour of $v$. Let $c$ be the other neighbour of $w$. If $a=c$, then $G=K_{5} \backslash e$, in which case $G$ is not a counterexample by Lemma 6.2. Thus $a \neq c$, and $(a, b, c, d)$ is a 4-cycle whose interior only contains $v$ and $w$.

At least one of $a c$ and $b d$ is not an edge of $G$, as otherwise $G$ would contain a subdivision of $K_{5}$. If $a c$ is not an edge of $G$, then let $G^{\prime}$ be the graph obtained from $G$ by deleting $v$ and $w$, and adding the edge $a c$. Otherwise, let $G^{\prime}$ be the graph obtained from $G$ by deleting $v$ and $w$, and adding the edge $b d$. In both cases, $G^{\prime}$ is a triangulation on $n-2$ vertices. Since $G$ is minimum, $G^{\prime}$ is not a counterexample. Thus $G^{\prime}$ has a flippable set $S^{\prime}$ of at least $\frac{1}{3}(n-4)$ edges. Initialise $S:=S^{\prime}$.

First suppose that $a c$ is not an edge of $G$. Then $a c$ is an edge of $G^{\prime}$. If $a b \in S^{\prime}$, then let $S:=S \cup\{w b\}$. If $b c \in S^{\prime}$, then let $S:=S \cup\{v b\}$. If $c d \in S^{\prime}$, then let $S:=S \cup\{v d\}$. If $a d \in S^{\prime}$, then let $S:=S \cup\{w d\}$.

Now suppose that $a c$ is an edge of $G$. Then $b d$ is an edge of $G^{\prime}$. If $a b \in S^{\prime}$, then let $S:=S^{\prime} \cup\{v d\}$. If $a d \in S^{\prime}$, then let $S:=S^{\prime} \cup\{v b\}$. If $c d \in S^{\prime}$, then let $S:=S^{\prime} \cup\{w b\}$. If $b c \in S^{\prime}$, then let $S:=S^{\prime} \cup\{w d\}$.

If none of these cases occur, then let $S:=S \cup\{v b, w d\}$. If both $v b$ and $v d$ have been added to $S$, then delete one from $S$. If both $w b$ and $w d$ have been added to $S$, then delete one from $S$. It is easily seen that in each case, $S$ is a flippable set, and $|S| \geq\left|S^{\prime}\right|+1 \geq \frac{1}{3}(n-4)+1>\frac{1}{3}(n-2)$. Thus $G$ is not a counterexample.

The following lemma is the key idea in the proof of Theorem 6.1

Lemma 6.5. Let $\left\{E_{1}, E_{2}, E_{3}\right\}$ be an edge 3-colouring of a triangulation $G$ such that every triangle is trichromatic. For each $1 \leq i \leq 3$, let $S_{i}$ be the set of edges in $E_{i}$ that are not in a bad pair with some other edge in $E_{i}$. Then $S_{i}$ is flippable in $G$.

Proof. Since every triangle is trichromatic, no two edges in $S_{i}$ are consecutive. This is condition (1) in Lemma 2.1. Condition (2) in Lemma 2.1 holds by the definition of $S_{i}$. Suppose that an edge $a b \in S_{i}$ is blocked by an edge $v w$. To show that condition (3) of Lemma 2.1 is satisfied, we need to prove that $v w \in S_{i}$.

First suppose that $v w \notin E_{i}$. Since $(v, a, w)$ is a triangle, one of $a v$ and $b v$ is in $E_{i}$, which implies that this edge and $a b$ are consecutive and both in $E_{i}$. This contradiction proves that $v w \in E_{i}$. Now suppose that $v w$ and some edge $x y$ form a bad pair. By Lemma 2.5 $v w$ and $x y$ are in a common triangle. Thus $x y \notin E_{i}$ and $v w$ does not form a bad pair with another edge in $E_{i}$. Therefore $v w \in S_{i}$, as desired. By Lemma 2.1, $S_{i}$ is flippable.

An edge is bad if it is a member of a bad pair. An edge is good if it is not bad.

Lemma 6.6. If every edge in a face $(u, v, w)$ of a triangulation $G$ is bad, then at least one of $\{u, v, w\}$ has degree three or four. 
Proof. Assume $\operatorname{deg}(u) \leq \operatorname{deg}(v) \leq \operatorname{deg}(w)$. If $\operatorname{deg}(u)=3$ then we are done. Suppose that $\operatorname{deg}(u) \geq 4$. Let $x, y, z$ be the other vertices that respectively see the edges $u v, v w, u w$. Since each of $u, v, w$ have degree at least four, $x, y, z$ are distinct. As illustrated in Figure 9 with an outerface of $(u, v, w)$, there are edges $a b, c d$, and ef such that $\{u v, a b\},\{u w, e f\},\{v w, c d\}$ are all bad pairs. For planarity to hold, and since $\operatorname{deg}(u) \leq \operatorname{deg}(v) \leq \operatorname{deg}(w), d=x$ and $c=z$, which implies that $\operatorname{deg}(u)=4$, as desired.

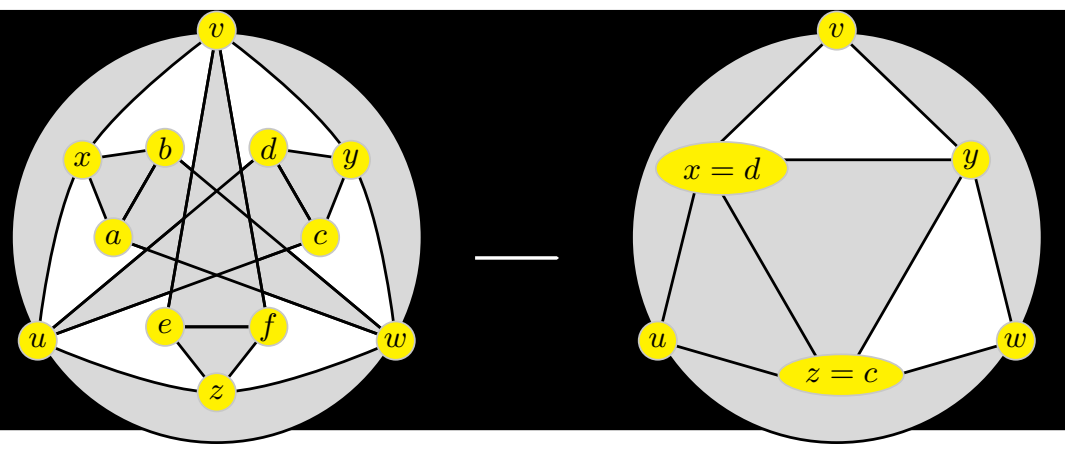

Figure 9: Three bad edges $u v, u w$, and $v w$ all on one face.

Lemma 6.7. Define $S_{1}, S_{2}, S_{3}$ as in Lemma 6.5 Then every edge in a separating triangle is in $S_{1} \cup S_{2} \cup S_{3}$.

Proof. Consider an edge $v w \in E_{i}$ that is in a separating triangle $T$. If $v w$ is good then $v w \in S_{i}$ and we are done. Otherwise $v w$ is bad. By Lemma 2.3, the edge $e$ that forms a bad pair with $v w$ is also in $T$. Since each triangle is trichromatic, $e \notin E_{i}$. Thus $v w \in S_{i}$.

Lemma 6.8. In a minimum counterexample, every edge seen by a degree-4 vertex is good.

Proof. Let $v$ be a degree- 4 vertex in a minimum counterexample $G$. Let $(a, b, c, d)$ be the neighbours of $v$ in cyclic order. Then $X:=\{a b, b c, c d, a d\}$ are the edges seen by $v$. Suppose on the contrary that one edge in $X$, say $a d$, is bad. Then $a d$ forms a bad pair with another edge in $X$. Without loss of generality, either $\{a b, a d\}$ or $\{a d, b c\}$ are this bad pair. If $\{a b, a d\}$ is a bad pair, then to avoid parallel edges, $\operatorname{deg}(a)=4$, which contradicts Lemma 6.4

Now suppose that $\{a d, b c\}$ is a bad pair. Let $x$ be the other vertex seen by these edges. Let $G^{\prime}$ be the plane graph obtained from $G$ by deleting $v$, deleting the edges in the triangle $(c, d, x)$, merging the vertices $a$ and $d$, and merging the vertices $b$ and $c$. Then $G^{\prime}$ is a triangulation on $n-3$ vertices. Since $G$ is minimum, $G^{\prime}$ is not a counterexample. Thus $G^{\prime}$ has a flippable set $S^{\prime}$ of at least $\frac{1}{3}(n-5)$ edges. Let $S:=S^{\prime} \cup\{v d\}$. We claim that $S$ is flippable in $G$. Now $v d$ flips to $a c$, which is not an edge of $G$ as otherwise there would be a subdivision of $K_{5}$. The only edge that forms a bad pair with $v d$ is $v b$, which by construction is not in $S$. Thus $S$ is flippable, and $|S|=\left|S^{\prime}\right|+1 \geq \frac{1}{3}(n-5)+1=\frac{1}{3}(n-2)$. Thus $G$ is not a counterexample.

Proof of Theorem 6.1 Let $G$ be a minimum counterexample with $n$ vertices. By Lemma 3.9 there is a 3 -colouring $\left\{E_{1}, E_{2}, E_{3}\right\}$ of the edges of $G$ such that every triangle is trichromatic. Let $S_{i}$ be set of edges in $E_{i}$ that are not in a bad pair with another edge in $E_{i}$. By Lemma 6.5 $S_{i}$ is flippable. 
The neighbours of a degree-3 vertex form a separating triangle. By Lemma 6.7 every face incident to a degree-3 vertex has at least one edge in $S_{1} \cup S_{2} \cup S_{3}$. By Lemma 6.8, every face incident to a degree- 4 vertex has one good edge, which is in $S_{1} \cup S_{2} \cup S_{3}$. By Lemma 6.6 every face not incident to degree- 3 or degree- 4 vertex has at least one good edge, which is in $S_{1} \cup S_{2} \cup S_{3}$.

We conclude that every face has at least one edge in $S_{1} \cup S_{2} \cup S_{3}$. There are $2(n-2)$ faces and every edge is in two faces. Thus $\left|S_{1} \cup S_{2} \cup S_{3}\right| \geq n-2$. For some $i$, we have $\left|S_{i}\right| \geq \frac{1}{3}(n-2)$. Therefore $G$ is not a counterexample, and since $G$ was minimum, there are no counterexamples.

Now for some upper bounds on $\operatorname{msf}(G)$.

Lemma 6.9. For every $n$-vertex triangulation $G, \operatorname{msf}(G) \leq n-2$.

Proof. Let $S$ be a flippable set of edges of $G$. Every edge in $S$ is incident to two distinct faces, and no other edge on each of these faces is in $S$. (Otherwise there would be two consecutive edges in $S$.) There are $2(n-2)$ faces in a triangulation. Thus $|S| \leq n-2$.

Lemma 6.10. There exist an $n$-vertex triangulation $G$ with $\operatorname{msf}(G)=\frac{6}{7}(n-2)$ for infinitely many $n$.

Proof. Let $G_{0}$ be an arbitrary triangulation with $n_{0}$ vertices. Let $G$ be the triangulation obtained from $G_{0}$ by adding a triangle inside each face $(u, v, w)$ of $G$, each vertex of which is adjacent to two of $\{u, v, w\}$. Say $G$ has $n$ vertices. Then $n-2=n_{0}+3\left(2 n_{0}-4\right)-2=7\left(n_{0}-2\right)$. Let $S$ be a flippable set of edges of $G$.

There is at most one edge in $S$ on the boundary of each face of $G$. Suppose on the contrary that for some face $(u, v, w)$ of $G_{0}$, all seven of the corresponding faces of $G$ have an edge in $S$. Every edge in $S$ is on the boundary of two faces of $G$. Thus $|S \cap\{u v, u w, v w\}|=1$ or 3 . Let $(x, y, z)$ be the triangle of $G$ inside $(u, v, w)$, with connecting edges $\{x v, x w, y u, y w, z u, z v\}$.

Case 1. $|S \cap\{u v, u w, v w\}|=1$ : Without loss of generality $S \cap\{u v, u w, v w\}=\{u v\}$, as illustrated in Figure 10(a) and (b). Thus either (a) $u y \in S$ or (b) $z y \in S$. If $u y \in S$, then $x y \notin S$ (as otherwise $G\langle S\rangle$ would have parallel edges). Thus $\{x z, x w\} \in S$, in which case $G\langle S\rangle$ has parallel edges, a contradiction. If $z y \in S$, then $y w \in S$, as otherwise no edge on $(u, w, y)$ would be in $S$. In this case $G\langle S\rangle$ has parallel edges.

Case 2. $|S \cap\{u v, u w, v w\}|=3$ : Then $z y$ is the only edge on the boundary of the face $(u, z, y)$ that can be flipped, as illustrated in Figure 10(c). Hence $z y \in S$. This implies that no edge on the faces $(z, v, x)$ and $(x, y, w)$ can be flipped, a contradiction.

Therefore for every face of $G_{0}$, at least one of the seven corresponding faces of $G$ does not have an edge in $S$. Hence at least $2\left(n_{0}-2\right)=\frac{2}{7}(n-2)$ faces of $G$ do not have an edge in $S$. Every face of $G$ has at most one edge in $S$. Thus $|S| \leq \frac{1}{2}\left(2(n-2)-\frac{2}{7}(n-2)\right)=\frac{6}{7}(n-2)$.

It remains to construct a flippable set of $\frac{6}{7}(n-2)$ edges in $G$. For each face of $G_{0}$, add the edges shown in Figure 10(d) to a set $S$. Clearly $S$ is flippable. In every face of $G_{0}$, exactly one of the corresponding seven faces of $G$ does not have an edge in $S$, and the remaining six faces each have exactly one edge in $S$. By the above analysis, $|S|=\frac{6}{7}(n-2)$.

An obvious open problem is to close the gap between the lower bound of $\frac{1}{3}(n-2)$ and the upper bound of $\frac{6}{7}(n-2)$ in the above results. For 5 -connected triangulations we can improve the lower bound as follows. 


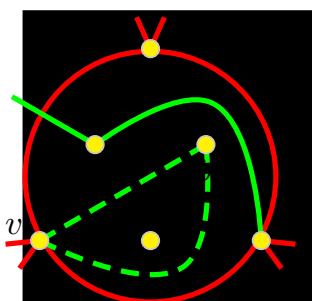

(a)

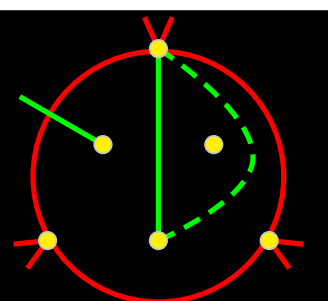

(b)

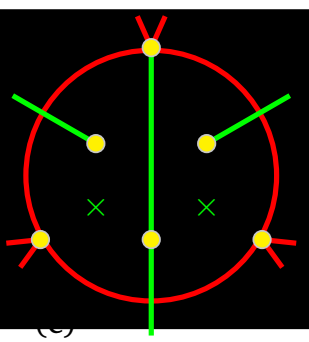

(c)

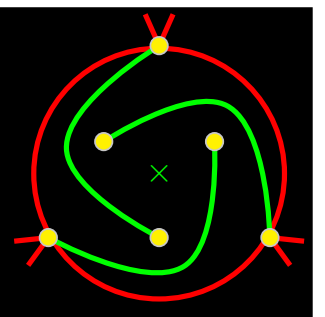

(d)

Figure 10: (a)-(c) For any number of flips in the outer triangle, at least one internal face does not have an edge in $S$. (d) How to construct a flip set for $G$.

Theorem 6.11. For every 5-connected triangulation $G$ with $n$ vertices, $\operatorname{msf}(G)=n-2$.

Proof. Observe that every edge in $G$ is flippable, as otherwise $G$ has a separating triangle (since $G$ has at least five vertices). There is no bad pair in $G$, as otherwise $G$ has a separating 4-cycle. By Lemma 2.1 a set of edges $S$ in a 5-connected triangulation $G$ is flippable if and only if no two edges in $S$ are consecutive. By Lemma 3.4 $G$ has a set of edges $S$ such that every triangle of $G$ has exactly one edge in $S$. Thus no two edges in $S$ are consecutive. Hence $S$ is flippable. By the argument employed in Lemma 6.9. $|S|=n-2$. Therefore $\operatorname{msf}(G) \geq n-2$. By Lemma 6.9. $\operatorname{msf}(G) \leq n-2$.

\section{Acknowledgements}

The research of Prosenjit Bose was partially completed at the Departament de Matemàtica Aplicada II, Universitat Politècnica de Catalunya, Barcelona, Spain. Thanks to Ferran Hurtado for graciously hosting the visit. Thanks to the referees for pointing out an error in a preliminary version of the paper.

\section{References}

[1] Therese C. Biedl, Prosenjit Bose, Erik D. Demaine, and Anna Lubiw. Efficient algorithms for Petersen's matching theorem. J. Algorithms, 38(1):110-134, 2001.

[2] Prosenjit Bose, Vida Dujmović, And David R. Wood. Induced subgraphs of bounded treewidth and bounded degree. Contrib. Discrete Math., 1(1):88-105, 2006.

[3] Richard Brunet, Atsuhiro Nakamoto, and Seiya Negami. Diagonal flips of triangulations on closed surfaces preserving specified properties. J. Combin. Theory Ser. B, 68(2):295-309, 1996.

[4] Yen-Ju Chen, Jou-Ming Chang, And Yue-Li Wang. An efficient algorithm for estimating rotation distance between two binary trees. Int. J. Comput. Math., 82(9):1095-1106, 2005.

[5] Norishige Chiba And TAKao Nishizeki. The Hamiltonian cycle problem is linear-time solvable for 4-connected planar graphs. J. Algorithms, 10(2):187-211, 1989. 
[6] Marek Chrobak and Thomas H. Payne. A linear-time algorithm for drawing a planar graph on a grid. Inform. Process. Lett., 54(4):241-246, 1995.

[7] Carmen Cortés, Clara Grima, Alberto Marquez, and Atsuhiro NaKamoto. Diagonal flips in outer-triangulations on closed surfaces. Discrete Math., 254(1-3):63-74, 2002.

[8] Carmen CortÉs And Atsuhiro NaKAmoto. Diagonal flips in outer-torus triangulations. Discrete Math., 216(1-3):71-83, 2000.

[9] Hubert de Fraysseix, János Pach, and Richard Pollack. How to draw a planar graph on a grid. Combinatorica, 10(1):41-51, 1990.

[10] Jerôme Galtier, Ferran Hurtado, Marc Noy, Stephane Pérennes, and Jorge UrRUTIA. Simultaneous edge flipping in triangulations. Internat. J. Comput. Geom. Appl., 13(2):113-133, 2003.

[11] Zhicheng GaO, Jorge Urrutia, and Jianyu Wang. Diagonal flips in labelled planar triangulations. Graphs Combin., 17(4):647-657, 2001.

[12] Zhicheng GAO AND JiANYU WANG. Enumeration of rooted planar triangulations with respect to diagonal flips. J. Combin. Theory Ser. A, 88(2):276-296, 1999.

[13] Sabine Hanke, Thomas Ottmann, and Sven Schuierer. The edge-flipping distance of triangulations. J.UCS, 2(8):570-579, 1996.

[14] Ferran Hurtado and Marc Noy. Graph of triangulations of a convex polygon and tree of triangulations. Comput. Geom., 13(3):179-188, 1999.

[15] Ferran Hurtado, Marc noy, and Jorge Urrutia. Flipping edges in triangulations. Discrete Comput. Geom., 22(3):333-346, 1999.

[16] Hideo Komuro. The diagonal flips of triangulations on the sphere. Yokohama Math. J., 44(2):115-122, 1997.

[17] Hideo Komuro And KiYoshi Ando. Diagonal flips of pseudo triangulations on the sphere. Ars Combin., 59:225-239, 2001.

[18] Hideo Komuro, Atsuhiro Nakamoto, and Seiya Negami. Diagonal flips in triangulations on closed surfaces with minimum degree at least 4. J. Combin. Theory Ser. B, 76(1):68-92, 1999.

[19] DÉNES KÖNIG. Theorie der endlichen und unendlichen Graphen. Kombinatorische Topologie der Streckenkomplexe. Akademische Verlagsgesellschaft, Leipzig, 1936.

[20] Ryuichi Mori, Atsuhiro Nakamoto, and Katsuhiro Ota. Diagonal flips in Hamiltonian triangulations on the sphere. Graphs Combin., 19(3):413-418, 2003.

[21] Томокі NaKAmigaWA. A generalization of diagonal flips in a convex polygon. Theoret. Comput. Sci., 235(2):271-282, 2000.

[22] Atsuhiro Nakamoto And Seiya Negami. Diagonal flips in graphs on closed surfaces with specified face size distributions. Yokohama Math. J., 49(2):171-180, 2002. 
[23] Atsuhiro Nakamoto, Tadashi Sakuma, and Yusuke Suzuki. $N$-flips in even triangulations on the sphere. J. Graph Theory, 51(3):260-268, 2006.

[24] SeiYa Negami. Diagonal flips in triangulations of surfaces. Discrete Math., 135(1-3):225$232,1994$.

[25] SeiYa Negami. Diagonal flips in triangulations on closed surfaces, estimating upper bounds. Yokohama Math. J., 45(2):113-124, 1998.

[26] SeiYa Negami. Diagonal flips of triangulations on surfaces, a survey. Yokohama Math. J., 47:1-40, 1999.

[27] Seiya Negami and Atsuhiro Nakamoto. Diagonal transformations of graphs on closed surfaces. Sci. Rep. Yokohama Nat. Univ. Sect. I Math. Phys. Chem., 40:71-97, 1993.

[28] Jean Pallo. On the rotation distance in the lattice of binary trees. Inform. Process. Lett., 25(6):369-373, 1987.

[29] JEAn Pallo. An efficient upper bound of the rotation distance of binary trees. Inform. Process. Lett., 73(3-4):87-92, 2000.

[30] Julius Petersen. Die Theorie der regulären Graphen. Acta. Math., 15:193-220, 1891.

[31] Neil Robertson, Daniel P. Sanders, Paul D. Seymour, and Robin Thomas. The fourcolour theorem. J. Combin. Theory Ser. B, 70(1):2-44, 1997.

[32] Daniel D. Sleator, Robert E. Tarjan, and William P. Thurston. Rotation distance, triangulations, and hyperbolic geometry. J. Amer. Math. Soc., 1(3):647-681, 1988.

[33] Daniel D. Sleator, Robert E. Tarjan, and William P. Thurston. Short encodings of evolving structures. SIAM J. Discrete Math., 5(3):428-450, 1992.

[34] Peter Guthrie Tait. Note on a theorem in geometry of position. Trans. Roy. Soc. Edinburgh, 29:657-660, 1880.

[35] Klaus Wagner. Bemerkung zum Vierfarbenproblem. Jber. Deutsch. Math.-Verein., 46:2632, 1936.

[36] Takahiro Watanabe And Seiya Negami. Diagonal flips in pseudo-triangulations on closed surfaces without loops. Yokohama Math. J., 47:213-223, 1999.

[37] Hassler Whitney. A theorem on graphs. Ann. of Math. (2), 32(2):378-390, 1931. 